\title{
Data Mining of Coherent Structures from Turbulence Tomographic TR-PIV Signal Database Utilizing Improved Quadrant Splitting Method
}

\author{
Shaoqiong Yang ${ }^{2,3}$, Yifan $\mathrm{Li}^{2}$ \\ ${ }^{2}$ Department of Mechanics, \\ Tianjin University, \\ Tianjin, China \\ shaoqiongy@tju.edu.cn
}

\author{
Nan Jiang ${ }^{1,2,3}$ \\ ${ }^{1}$ State Key Laboratory of Non-linear Mechanics (LNM), \\ Institute of Mechanics, Chinese Academy of Sciences, \\ Beijing, China \\ ${ }^{3}$ Tianjin Key Laboratory of Modern Engineering \\ Mechanics, Tianjin, China
}

\begin{abstract}
In this paper, we calculated the spatial locally averaged velocity strains along the streamwise direction with four scales in the velocity vector field of turbulent boundary layer by utilizing the three-dimensional three-component database of time series of velocity field measured by tomographic time-resolved particle image velocimetry. We introduced an improved quadrant splitting method based on the spatial local-averaged velocity strains and a new conditional sampling phase average method as a datamining criterion to detect and extract the inherent coherent structures from such turbulence signal database. Furthermore, we used the improved quadrant splitting method to obtain and investigate the spatial topologies of fluctuating velocity and fluctuating vorticity with four scales whose center was a strong second-quadrant event (Q2) or a fourth-quadrant event (Q4). Results illustrate that there is a better similarity of the multiscale coherent structures in wall-normal direction than the one in other two dimensions.
\end{abstract}

Keywords-data mining; turbulent boundary layer; TR-PIV; coherent structure; Q2/Q4; local-averaged velocity strain; IQSM

\section{INTRODUCTION}

In recent years, with the rapid development of threedimensional particle image velocimetry (3D-PIV) technology, the data mining (detection and extraction [1]) of threedimensional coherent structures(CSs) of turbulent boundary layers(TBLs) utilizing relevant experimental technology and data processing algorithms[2-3] as well as the analysis of their topology characteristics become a research focus. The coherent structures are defined as such structures that possess the same flow characteristics or closely relate to each other within a space whose spatial magnitude is in the order of boundary layer thickness. These CSs have significant influences on the skin friction, mass and heat transfer within the TBLs, and on the generation of turbulence kinetic energy as well. The induced velocities of vortical structures influence the evolution of other CSs, thus they are the kernels of the CSs in a TBL.[4] Burst created by the low-speed fluid ejecting from wall region (also called Q2, namely ejection event) or by the high-speed fluid sweeping towards the wall (Q4 or sweep event) is the most important source for the generation of turbulent energy and Reynolds shear stress.[1,5] Existing research results show that within the TBLs we can clearly observe the repeatability [6] of a variety of similar processes as well as the extended selfsimilarity [7] of multi-scale eddy structures. In this paper, the spatial locally averaged velocity strains along the streamwise direction with four scales in the velocity field of turbulent boundary layer were calculated according to the conception of spatial local-averaged velocity structure function by using the three-dimensional three-component (3D-3C) database of time series of velocity vector field measured by tomographic timeresolved PIV (TR-PIV). An improved quadrant splitting method (IQSM) based on the spatial local-averaged velocity strains together with a new conditional sampling phase average method was introduced as a data mining criterion to detect and extract the inherent coherent structures in the TBL. Furthermore, we used the IQSM to obtain and investigate the spatial topologies of fluctuating velocity and fluctuating vorticity with four scales whose center being a strong Q2 event or a Q4 event.

\section{DATABASES AND DATA MinING METHODS}

The databases of the TBL with zero-pressure-gradient used in this paper have been obtained from a tomographic TR-PIV experiment in the water tunnel of the Aero and Hydrodynamics Laboratories of TU Delft. The setup and several first interesting results have been described in [8] and [9]. For completeness, however, we briefly recall some properties of the TBL whose free-stream flow velocity $U$ at $530 \mathrm{~mm} / \mathrm{s}$ here given below in Table 1. Please note that we have already made several appropriate additions and amendments when quoting existing excellent results from [8]. More details can be found in [10]. Here $R e_{\theta}$ is the Reynolds number based on momentum thickness and free-stream velocity. The friction Reynolds number $R e_{\tau}=u_{\tau} \delta / v$ and $v$ is the kinematic viscosity. The TBL databases to be processed totally consist of 5 groups, for each group containing 1023 instantaneous 3D-3C velocity vector volumes. It is over a grid of $92 \times 99 \times 22$ (streamwise $\times$ spanwise $\times$ normal, totally 200376) measurement points located every $0.687 \mathrm{~mm}$, corresponding to a spatial resolution of 15 wall units(WU) in all three directions in space for each time step of $2 \mathrm{~ms}$ increment. Fig. 1 illustrates one of original 3D-3C velocity vector fields with colors and vector arrows standing for velocity vector strength and direction respectively. We can consider it as a fuzzy system due to the randomness of such data signals.

This work was jointly funded by the National Basic Research Program of China (Grant No.2012CB720101), the National Natural Science Foundation of China (Grant No.10832001) and the Opening Subject of State Key Laboratory of Nonlinear Mechanics, Institute of Mechanics, Chinese Academy of Sciences. 
TABLE I. BASIC FLUID MeChanical Properties OF the TBL, AS ESTIMATED BY PIV

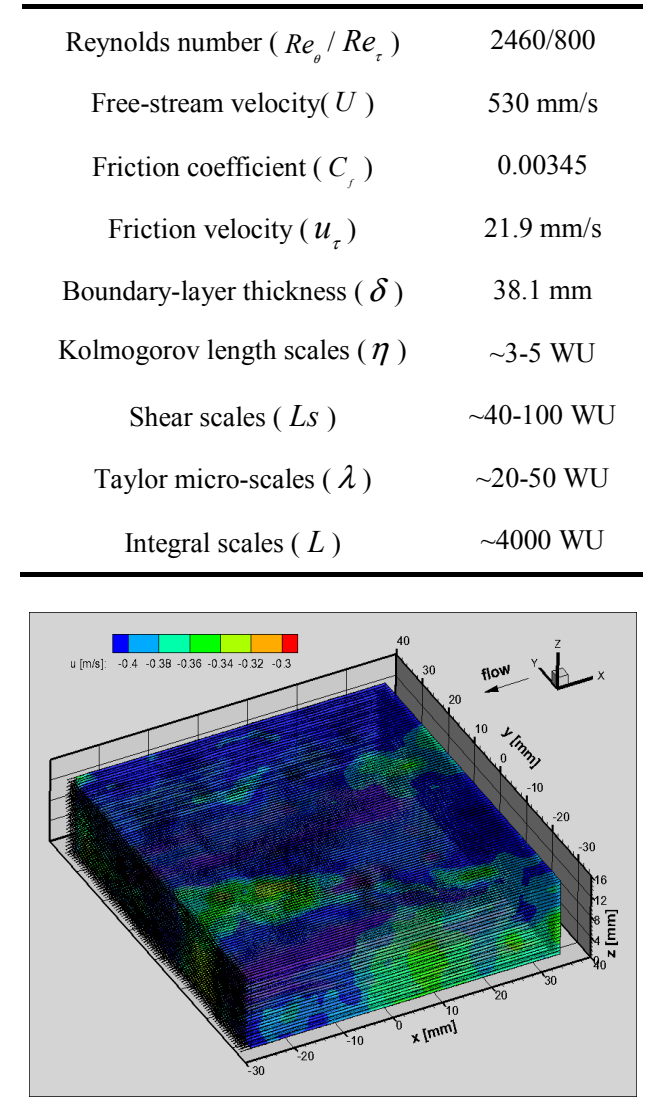

Figure 1. One of original 3D-3C velocity vector fields with colors and vector arrows standing for strength and sign respectively.

In turbulent flows, however, the existence of multi-scale coherent eddy structures has been verified by the increasing palmary results $[7,11-12]$ and the movement of fluid particles in the turbulent boundary layer is restricted by such coherent eddy structures, which leads to intermittency [13] of turbulent flows. Therefore, a multitude of analysis methods are proposed to analyze the turbulent signals that seem to be random but in fact made up of multi-scale structures. One of them is wavelet analysis that decomposes the analyzed signal in both the physical and the scale space by convoluting such signal with a wavelet that is the compact-supported analytic function [14].

A new method based on the locally averaged velocity structure function proposed by LIU Wei et al. [15] is used to take into account the multi-scale character of the turbulent structures. Actually, the experiment conducted by themselves in a wind tunnel and the following study from Liu J H et al. [16] have proven that the locally averaged velocity structure function is in agreement with the wavelet-coefficient structure function. TANG Zhan-Qi et al.[17] developed the localaveraged velocity structure function with various spatial scales to the 2D case when they analyze the 2D TR-PIV data signals. Accordingly, in the present study, the local-averaged velocity structure function will be extended into 3D case when the 3D$3 \mathrm{C}$ database of time series of spatial velocity field measured by tomographic TR-PIV is preprocessed. Hence we can obtain the spatial local-averaged velocity strain which is convincing- ly in accordance with the concept of wavelet-coefficient when Harr wavelet transforming in the streamwise direction as

$$
\begin{aligned}
& a_{11}=W_{x}^{x}(l, b)=\delta u_{x}\left(l, b_{x}\right) \\
& =\overline{u(x, y, z)}_{x \in\left[b_{x}, b_{x}+l\right]}-\overline{u(x, y, z)_{x \in\left[b_{x}-l, b_{x}\right]}} \\
& a_{21}=W_{x}^{y}(l, b)=\delta v_{x}\left(l, b_{y}\right) \\
& =\overline{v(x, y, z)_{x \in\left[b_{y}, b_{y}+l\right]}}-\overline{v(x, y, z)_{x \in\left[b_{y}-l, b_{y}\right]}} \\
& a_{31}=W_{x}^{z}(l, b)=\delta w_{x}\left(l, b_{z}\right) \\
& =\overline{w(x, y, z)_{x \in\left[b_{z}, b_{z}+l\right]}}-\overline{w(x, y, z)_{x \in\left[b_{z}-l, b_{z}\right]}}
\end{aligned}
$$

where $W_{x}^{x}(l, b), W_{x}^{y}(l, b)$, and $W_{x}^{z}(l, b)$ indicate respectively the streamwise( $-x)$, spanwise $(-y)$ and wall-normal $(-z)$ waveletcoefficients of wavelet transform along streamwise direction in a certain spatial turbulent scale $l$ at certain spatial location $b . \overline{u(x, y, z)}$ is the local-averaged streamwise velocity of the fluid motion in two adjacent eddies whose centers are located at $b_{x}+1 / 2$ and $b_{x}-l / 2$, respectively, thus the localaveraged streamwise velocity strain $\delta u_{x}\left(l, b_{x}\right)$ in physical reveal the tensile and compressive deformation of certain structures in turbulent flows. Similarly, the locally averaged spanwise velocity strain $\delta v_{x}\left(l, b_{x}\right)$ and wall-normal velocity strain $\delta w_{x}\left(l, b_{x}\right)$ are defined in (1).

There is a consensus that several certain relationship between coherent structures and Q2 or Q4 events do exist in the TBL. Accordingly, a new data mining method to detect and extract coherent structures can be illustrated as

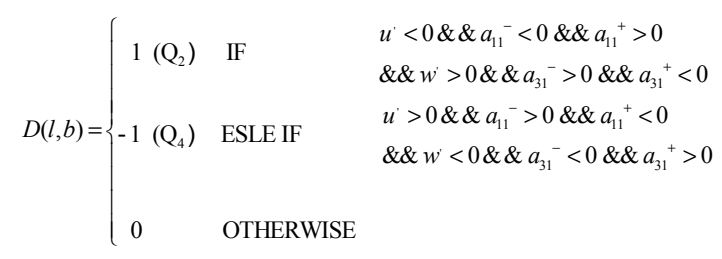

where $D(l, b)$ is the detection function in certain turbulent scale $l$ at certain location $b$, and $a_{11}{ }^{-}=\delta u_{x}\left(l, b_{x}\right)^{-}$is the left neighborhood value of streamwise local-averaged velocity strain at location $b_{x}$, while $a_{11}{ }^{+}$is the right neighborhood one. Formula (2) physically shows that Q2 events arise when $u^{\prime}<0, w^{\prime}>0$ and $u^{\prime}, w^{\prime}$ reach the local maximum and minimum respectively while Q4 events take place in $u^{\prime}>0, w^{\prime}<0$ and $u^{\prime}, w^{\prime}$ reach the local extremums respectively, as a result of which most importantly contribute to the Reynolds shear stress.[1, 18]

Given the quasi-periodic repeatability [6] of burst events and the extended self-similarity [7] of multi-scale eddy coherent structures, a new conditional sampling average method [19] based on the IQSM to extract turbulent coherent structures is given by

$$
\begin{aligned}
\left\langle f\left(l_{j}, x\right)\right\rangle_{Q_{2}} & =\left\{\frac{1}{N_{j}} \sum_{i=1}^{N_{j}} f\left(b_{i}+x\right) \mid-\frac{l_{j}}{2} \leq x \leq \frac{l_{j}}{2}, D\left(l_{j}, b_{i}\right)=1\right\} \\
\left\langle f\left(l_{j}, x\right)\right\rangle_{Q_{4}} & =\left\{\frac{1}{N_{j}} \sum_{i=1}^{N_{j}} f\left(b_{i}+x\right) \mid-\frac{l_{j}}{2} \leq x \leq \frac{l_{j}}{2}, D\left(l_{j}, b_{i}\right)=-1\right\}
\end{aligned}
$$




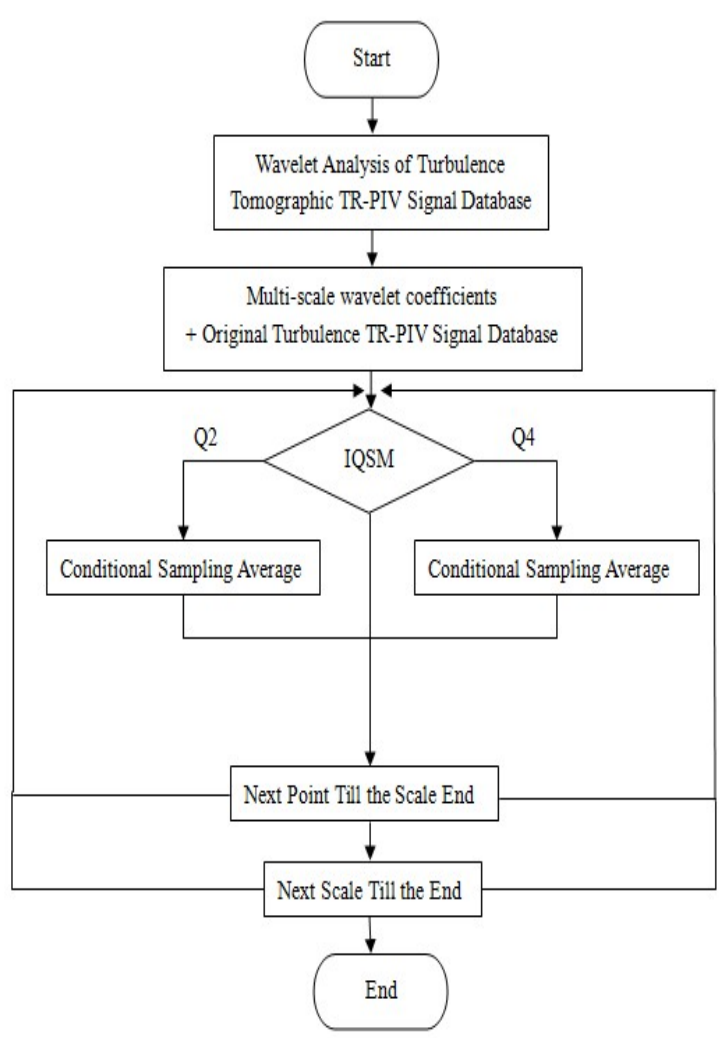

Figure 2. Data mining(detection and extraction) of multi-scale coherent structures in turbulent boundary layer

where \langle\rangle represents the ensemble average of certain variables, and $f\left(l_{j}, x\right)$ is the interesting conditional phase average component which respectively stands for fluctuating velocity, fluctuating vorticity etc. investigated in this present study. $N_{j}$ is the number of $j$-th scale of Q2 and Q4 events, while $l_{j}$ represents the duration of $j$-th scale of coherent structures. However, for convenient to compare, we cut uniformly 24 points out as values of $l_{j}$ along the streamwise and spanwise direction respectively, while $l_{j}=105 \mathrm{WU}$ along the wall-normal direction at every $j$-th scale. Fig.2 illustrates the program flow chart which can demonstrate how to detect and extract coherent structures from the turbulence tomographic TR-PIV signal database for data mining.

\section{RESULTS AND DISCUSSION}

In this paper, firstly, the spatial local-averaged velocity strains with four scales were calculated according to (1) along the streamwise direction by using the 3D-3C database of time series of velocity vector field measured by tomographic TRPIV. Here we need to point out that only four-scale localaveraged velocity strains were obtained owing to limits of insufficient data points in the wall-normal direction. Moreover, spatial phase average components that fluctuating velocity and fluctuating vorticity with four scales were obtained by cutting out then in alignment-superimposed phase averaging several

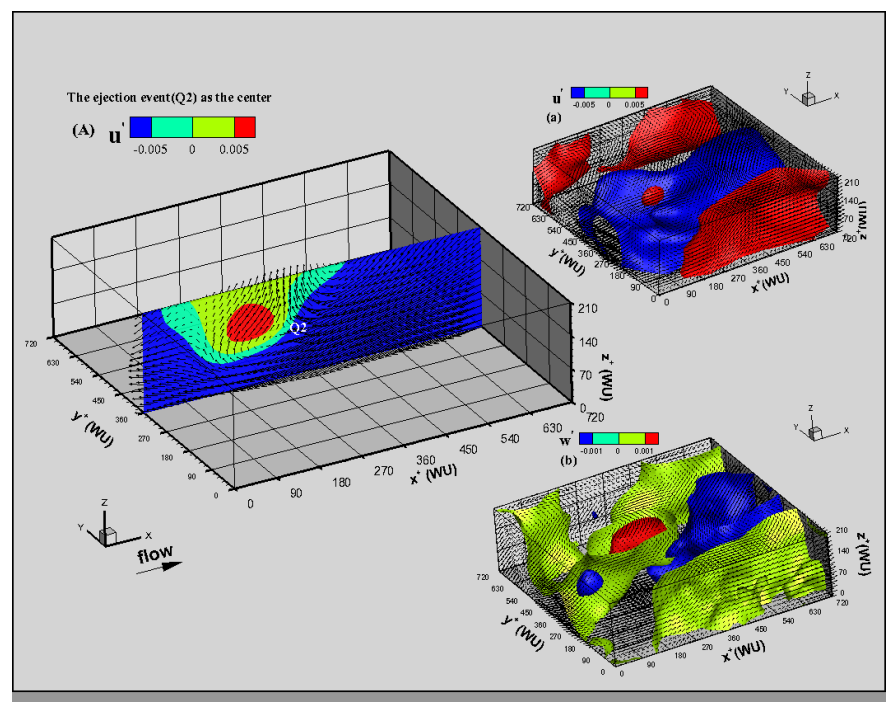

Figure 3. The fluctuating velocity distribution of coherent structures containing a Q2 event as the center. (A) the slice of streamwise velocity distribution with tangent velocity vectors at $\mathrm{y}^{+}=360 \mathrm{WU}$; (a) the iso-surface contours with three-level-value of streamwise fluctuating velocity distribution; (b) the iso-surface contours with three-level-value of wall-normal fluctuating velocity distribution.

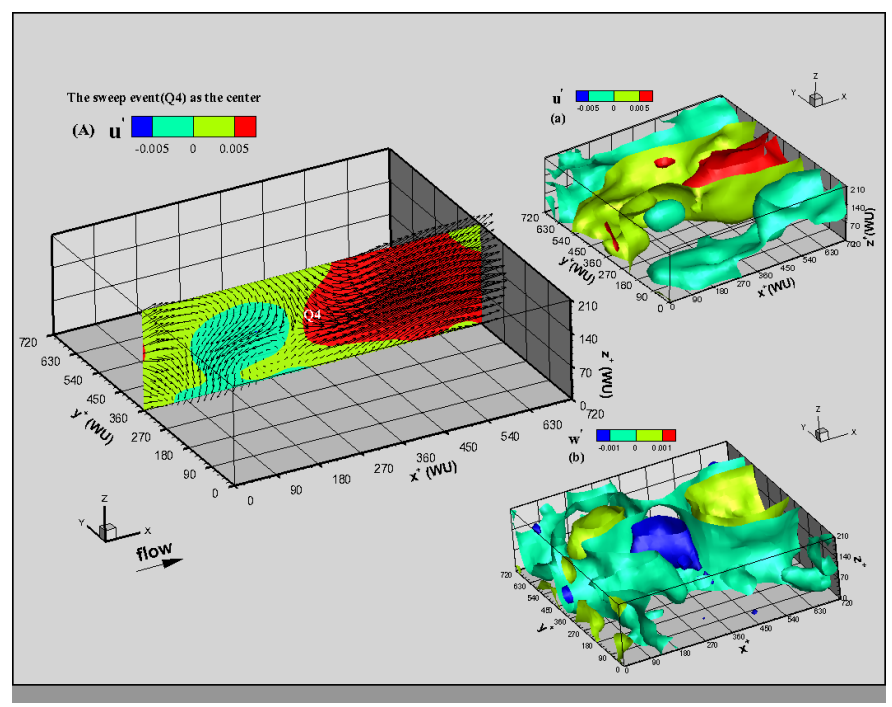

Figure 4. The fluctuating velocity distribution of coherent structures containing a Q4 event as the center. (A) the slice of streamwise velocity distribution with tangent velocity vectors at $\mathrm{y}^{+}=360 \mathrm{WU}$; (a) the iso-surface contours with three-level-value of streamwise fluctuating velocity distribution; (b) the iso-surface contours with three-level-value of wall-normal fluctuating velocity distribution.

rectangular volumes of $49 \times 49 \times 15$ measurement points, whose centers are in the spatial locations where they meet the condition (3) respectively, which, in other words, means that Q2 and Q4 bursting events are the centers of turbulent coherent structure spatial topology showed in Fig. 3 and Fig. 4. We can clearly find that the Q2 event arises together with a Q1 $\left(u^{\prime}>0, w^{\prime}>0\right)$ event contributing to the ejection process that makes the low-speed fluid ejected far away from the wall while the Q4 event together with a Q3 $\left(u^{\prime}<0, w^{\prime}<0\right)$ event makes the high-speed fluid swept into the low-speed region near the wall. In addition, in Fig.5 we give the iso-surface con- 


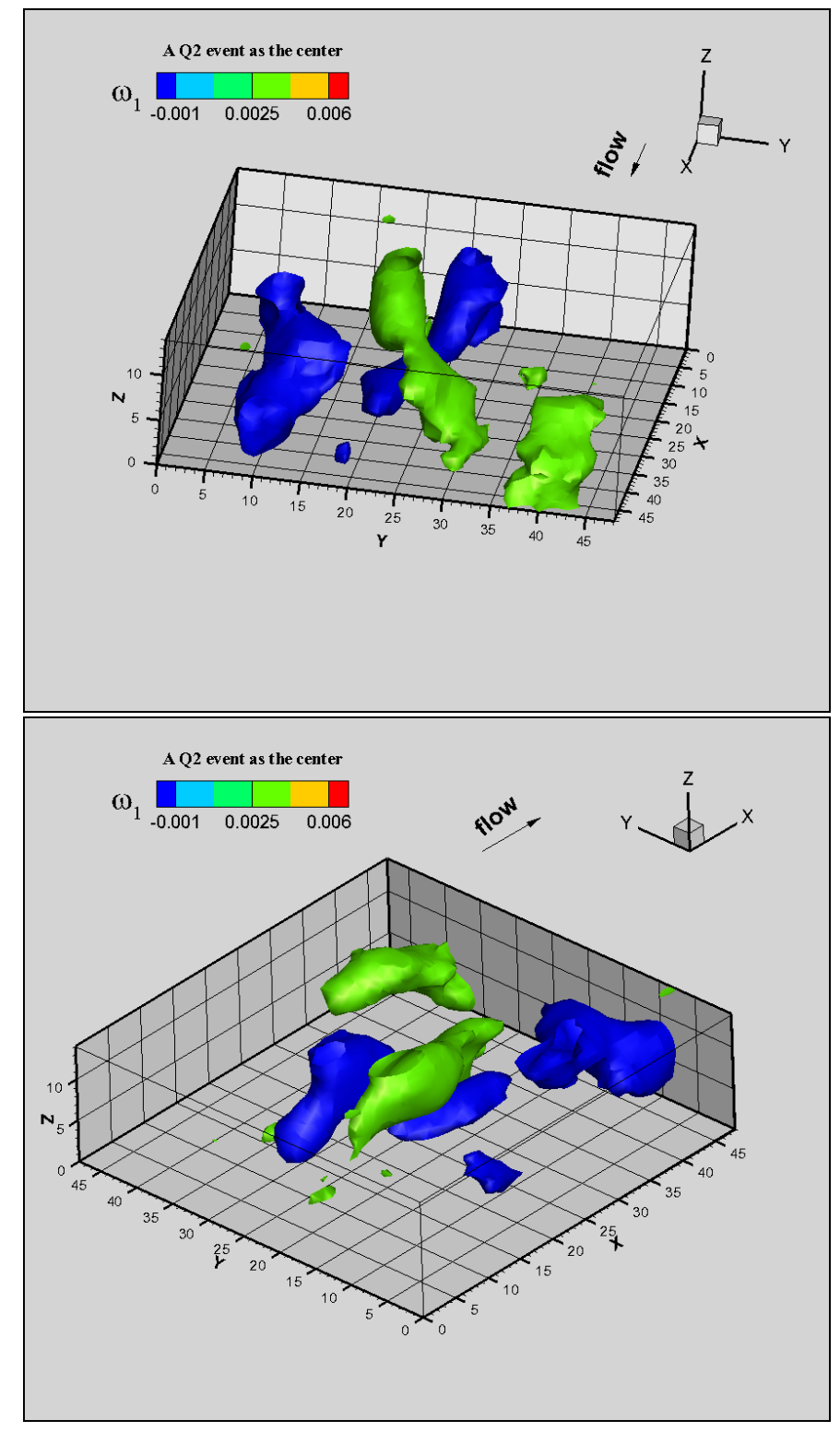

Figure 5. The iso-surface contours of streamwise fluctuating vorticity at the fourth scale from two different perspectives.

tour of streamwise fluctuating vorticity at the fourth scale from two different perspectives. The arrow shows the flow direction. It is clear that we have detected and extracted two couples of counter-rotating quasi-streamwise vortices which locates on both sides of the Q2 event but they change the sign and twist along the streamwise direction, thus it makes them into an "X chromosome" shape, which are the atypical coherent structures in the turbulent boundary layer. More specifically, we give a local enlarged contour in Fig.6 to illustrate details of these quasi-streamwise vortex pairs. Along the streamwise direction, upstream, the couple of coherent vortices labelled A and A' make the low-speed fluid (see Fig.3) of the near-wall region ejected into the outer region where they mix and exchange the mass and energy. After going through a singularity [20] which would not be disscussed here due to the length limit of this paper, they change their sign and

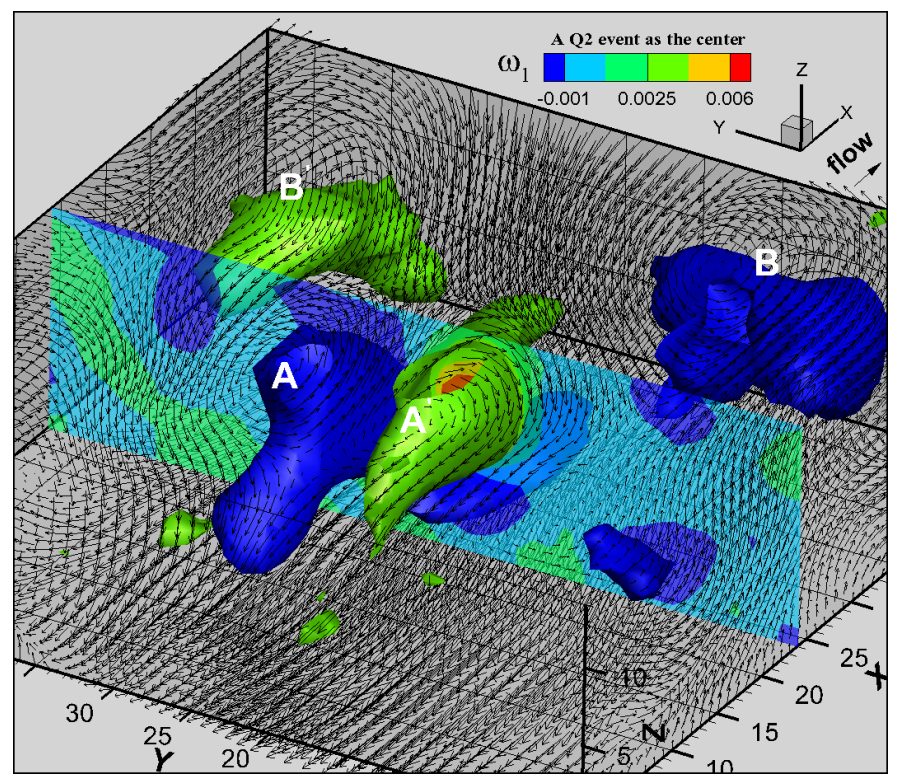

Figure 6. The local enlarged iso-surface contours of streamwise fluctuating vorticity with velocity vectors at the fourth scale containing a Q2 event as the center.

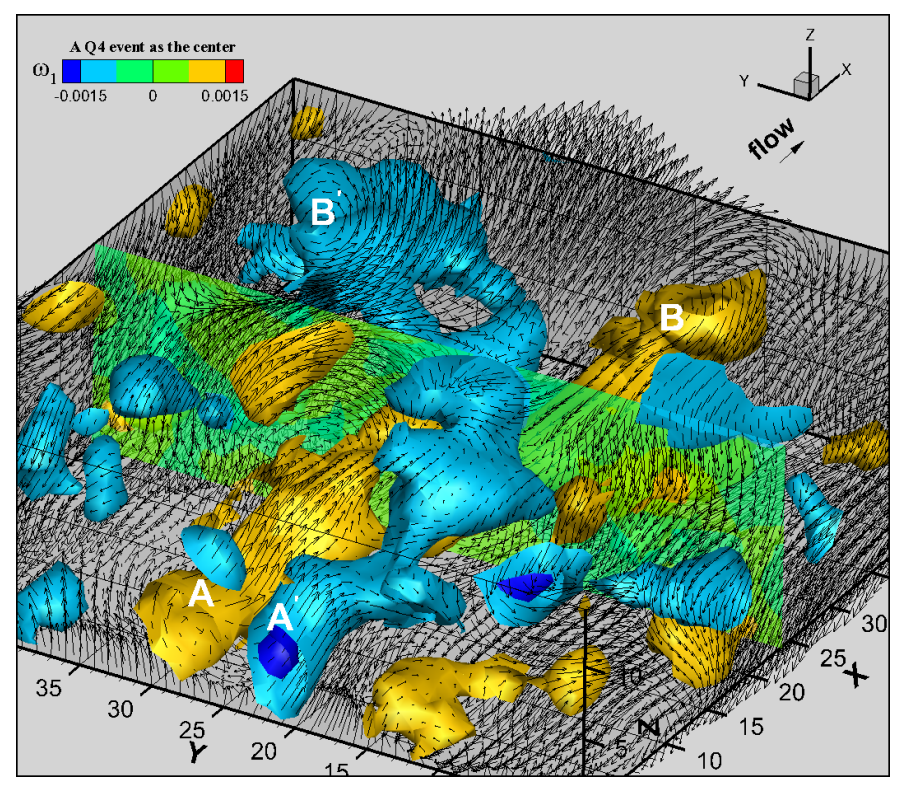

Figure 7. The local enlarged iso-surface contours of streamwise fluctuating vorticity with velocity vectors at the fourth scale containing a Q4 event as the center.

grow up in size while they make the high-speed fluid (see Fig.3) in outer region and both two sides swept towards the near-wall. Furthermore, for the completeness, we give a local enlarged iso-surface contour as well for srteamwise fluctuating vorticity with velocity vectors at the fourth scale when a $\mathrm{Q} 4$ event happens at the center in Fig.7. As can be seen, there are similar but opposite properties although such quasi-streamwise coherent vortices are more in number and even more complex in shape. If readers are interested in the properies of coherent 
structures or their more functions in the TBL, more interesting and excellent results you can find out from [1][4][11][20] ect.

\section{CONCLUSIONS}

Turbulent flow is made up of multi-scale eddy structures, and different coherent eddy structures exist at different scales. We have detected and extracted two couples of counter-rotating quasi-streamwise vortices with an "X chromosome" shape that locate on both sides of the Q2/Q4 event. According to the definition of Q2/Q4 bursting events as well as the results that we have detected and extracted from the tomographic TR-PIV velocity vector databases of the TBL, the IQSM and the new sample average method extended into the $3 \mathrm{D}-3 \mathrm{C}$ case are provenly applicable and effective for data-mining of coherent structures in the turbulent fuzzy system.

\section{ACKNOWLEDGMENT}

The authors are most grateful to the DLR Institute of Aerodynamics and Flow Technology for providing the tomographic TR-PIV database of time series of velocity vector field as well as the guidance and assistance of experimental technology for the present work.

\section{REFERENCES}

[1] G. Elsinga, D. Kuik, B. Van Oudheusden and F. Scarano, " Investigation of the three-dimensional coherent structures in a turbulent boundary layer with Tomographic-PIV", in 45th AIAA Aerospace Sciences Meeting and Exhibit, AIAA, Reno, Nevada, 2007, pp. 1305.

[2] G. E. Elsinga, F. Scarano, B. Wieneke and B. W. Van Oudheusden, "Tomographic particle image velocimetry," Experiments in Fluids, vol.41, pp. 933-947, 2006.

[3] G. E. Elsinga, "Tomographic particle image velocimetry and its application to turbulent boundary layers," Ph.D. dissertation, Delft University of Technology, 2008.

[4] Qixiang L. "Experimental studies on coherent structures in turbulent boundary layers," Advances in mechanics, 2006,36(3): 373-388.

[5] R. L. Panton, "Overview of the self-sustaining mechanisms of wall turbulence," Progress in Aerospace Sciences, vol.37, pp. 341-384, 2001.

[6] S. K. Robinson, "Coherent motions in the turbulent boundary layer," Annual Review of Fluid Mechanics, vol.23, pp. 601-639, 1991.
[7] S.Q. Yang and N. Jiang, "Wavelet analysis to detect multi-scale coherent eddy structures and intermittency in turbulent boundary layer" in Fuzzy Systems and Knowledge Discovery (FSKD), 2011 Eighth International Conference on, 2011, pp. 1241-1245.

[8] A. Schröder, R. Geisler, K. Staack, G. E. Elsinga, F. Scarano and B. Wieneke, et al., "Eulerian and Lagrangian views of a turbulent boundary layer flow using time-resolved tomographic PIV," Experiments in fluids, pp. 1-21, 2011.

[9] G. E. Elsinga and I. Marusic, "Evolution and lifetimes of flow topology in a turbulent boundary layer," Physics of Fluids, vol.22, pp. 15102, 2010.

[10] Yang S Q, Jiang N. "On the Measurement of Spatial Characteristic Scale in Turbulent Boundary Layer Based on Tomographic Time-resolved PIV". Journal of Experimental Mechanics, 2011, 26(04): 369-376.

[11] R. J. Adrian, C. D. Meinhart and C. D. Tomkins, "Vortex organization in the outer region of the turbulent boundary layer," Journal of Fluid Mechanics, vol.422, pp. 1-54, 2000.

[12] B. J. Balakumar and R. J. Adrian, "Large-and very-large-scale motions in channel and boundary-layer flows," Philosophical Transactions of the Royal Society A: Mathematical, Physical and Engineering Sciences, vol.365, pp. 665, 2007.

[13] J. Nan and Z. Jin, "Detecting multi-scale coherent eddy structures and intermittency in turbulent boundary layer by wavelet analysis," Chinese Physics Letters, vol.22, pp. 1968, 2005.

[14] M. Farge and K. Schneider, "Coherent vortex simulation (CVS), a semideterministic turbulence model using wavelets," Flow, Turbulence and Combustion, vol.66, pp. 393-426, 2001.

[15] L. Wei and J. Nan, "Three kinds of velocity structure function in turbulent flows," Chinese Physics Letters, vol.21, pp. 1989, 2004.

[16] L. Jian-hua, J. Nan, W. Zhen-dong and S. Wei, "Multi-scale coherent structures in turbulent boundary layer detected by locally averaged velocity structure functions," Applied Mathematics and Mechanics, vol.26, pp. 495-504, 2005.

[17] Z. Q. Tang and N. Jiang, "TR PIV Experimental Investigation on Bypass Transition Induced by a Cylinder Wake," Chinese Physics Letters, vol.28, pp. 54702, 2011.

[18] S. S. Lu and W. W. Willmarth, "Measurements of the structure of the Reynolds stress in a turbulent boundary layer," Journal of Fluid Mechanics, vol.60, pp. 481-511, 1973.

[19] Y. X. Jia, Z. Q. Tang and N. Jiang, "Experimental investigation of Reynolds stress complex eddy viscosity model for coherent structure dynamics," SCIENCE CHINA Physics, Mechanics \& Astronomy, pp. 1-9, 2011.

[20] S. Q. Yang and N. Jiang, "Tomographic TR-PIV measurement of coherent structure spatial topology utilizing an improved quadrant splitting method", unpublished. 